\title{
A modified gas-trapping method for high-throughput metabolic experiments in Drosophila melanogaster
}

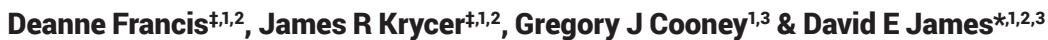

\begin{abstract}
Metabolism is often studied in animal models, with the Drosophila melanogaster fruit fly model offering ease of genetic manipulation and high-throughput studies. Fly metabolism is typically studied using end-point assays that are simple but destructive, and do not provide information on the utilization of specific nutrients. To address these limitations, we adapted existing gas-trapping protocols to measure the oxidation of radiolabeled substrates (such as glucose) in multi-well plates. This protocol is cost effective, simple, and offers precise control over experimental diet and measurement time, thus being amenable to high-throughput studies. Furthermore, it is nondestructive, enabling time-course experiments and multiplexing with other parameters. Overall, this protocol is useful for merging fly genetics with metabolic studies to understand whole organism responses to different macronutrients.
\end{abstract}

\section{METHOD SUMMARY}

We developed a gas-trapping protocol for studying metabolism in Drosophila melanogaster using perforated, mesh-covered plastic lids in sealed tissue-culture multiwell plates. This system enables precise control over experimental diet and time and is nondestructive, making it amenable to time-course experiments and multiplexing with other parameters.

\section{KEYWORDS}

carbon dioxide $\cdot$ Drosophila $\cdot$ gas-trap - metabolic labelling $\cdot$ metabolism

${ }^{1}$ Charles Perkins Centre, The University of Sydney, Camperdown, NSW 2006, Australia; ${ }^{2}$ School of Life \& Environmental Sciences, The University of Sydney, Camperdown, NSW 2006, Australia; ${ }^{3}$ School of Medical Sciences, The University of Sydney, Camperdown, NSW 2006, Australia; *Author for correspondence: david.james@ sydney.edu.au; ${ }^{\ddagger}$ These authors contributed equally to this work

BioTechniques 67: 123-125 (September 2019) 10.2144/btn-2019-0062
Over the past century, metabolism has been studied using a spectrum of models. On one end, in vitro cell-culture models are easily manipulatable and offer more control over the measurement of parameters in isolation. For instance, they are amenable to radiotracer studies to measure the oxidation of glucose in specific cell types, without being confounded by the presence of other cell types or substrates. In contrast, a more holistic view of metabolism can be obtained through assays at the wholeorganism level. These are frequently performed in rodent models [1,2], which are not readily genetically manipulated and often take longer than in vitro studies. A compromise between these two model systems is the fruit fly (Drosophila melanogaster). The fruit fly model has gained popularity in metabolic studies [3-6] because: 1) one can easily study large numbers of individuals in a short amount of time, thus it is amenable to highthroughput studies; 2) core metabolic pathways are conserved between flies and mammals [5]; 3) it is simple to knockdown and overexpress genes in flies [7]. Thus, the fruit fly is a live-animal model that offers mechanistic insight combined with whole-organism metabolism in the same experiments.

Current protocols for studying fly metabolism often involve end point measures of metabolites (i.e., fat, glycogen). These have several limitations, including being destructive and static (e.g., does fat content change due to increased storage or reduced utilization?), as well as not being able to provide information about specific nutrient utilization. To address this, we have developed a novel, nondestructive assay for measuring substrate oxidation in flies.

Fundamentally, this involves providing flies with a diet containing radiolabeled substrate (such as ${ }^{14} \mathrm{C}$-glucose), trapping any $\mathrm{CO}_{2}$ produced with alkali solution (as bicarbonate) and measuring radiation $\left({ }^{14} \mathrm{C}\right)$ in the trapped $\mathrm{CO}_{2}$. Similar protocols have pre-exposed flies to radiolabeled substrate (typically for 2-3 h), unlabeled substrate (for 2-4 h), then $\mathrm{CO}_{2}$ output measured in the absence of food (for 2-12 h) [8]. This methodology essentially measures residual substrate oxidation in the fasting state when other macronutrients such as fatty acids are probably being utilized [9]. Furthermore, such protocols require incubating flies in precut microfuge tubes sealed with mesh, within close proximity to alkali-soaked filtered paper (for $\mathrm{CO}_{2}$ collection). This apparatus is housed within $20-\mathrm{ml}$ scintillation vials sealed with a rubber stopper, which is not amenable to miniaturization for high-throughput studies. Thus, we sought to develop a protocol that was simple, cost effective and yet flexible in terms of when $\mathrm{CO}_{2}$ was collected relative to food intake (i.e., enabling substrate oxidation measurements in fed and/or fasted states).

To achieve this, we modified a gas-trapping method that we previously established for cell culture models [10]. This involved culturing cells in sealed multi-well plates, where $\mathrm{CO}_{2}$ was collected in a $\mathrm{NaOH}$ solution in a gas trap. The gas trap consisted of a perforated centrifuge tube lid which, when positioned upsidedown above the cells, allowed gaseous exchange through the hole in the lid while holding an $\mathrm{NaOH}$ solution in the inner rim of the lid. This enabled the assessment of substrate oxidation in a 12- or 24-well plate format, which would be ideal for highthroughput measurement of substrate oxidation in flies.

We adapted this gas-trapping method for flies through several modifications (Figure 1A-D). Diets containing radiolabeled substrate were prepared in multi-well plates prior to the experiment (Figure 1B). Anesthetized flies were then deposited into the wells. To ensure the $>$ 


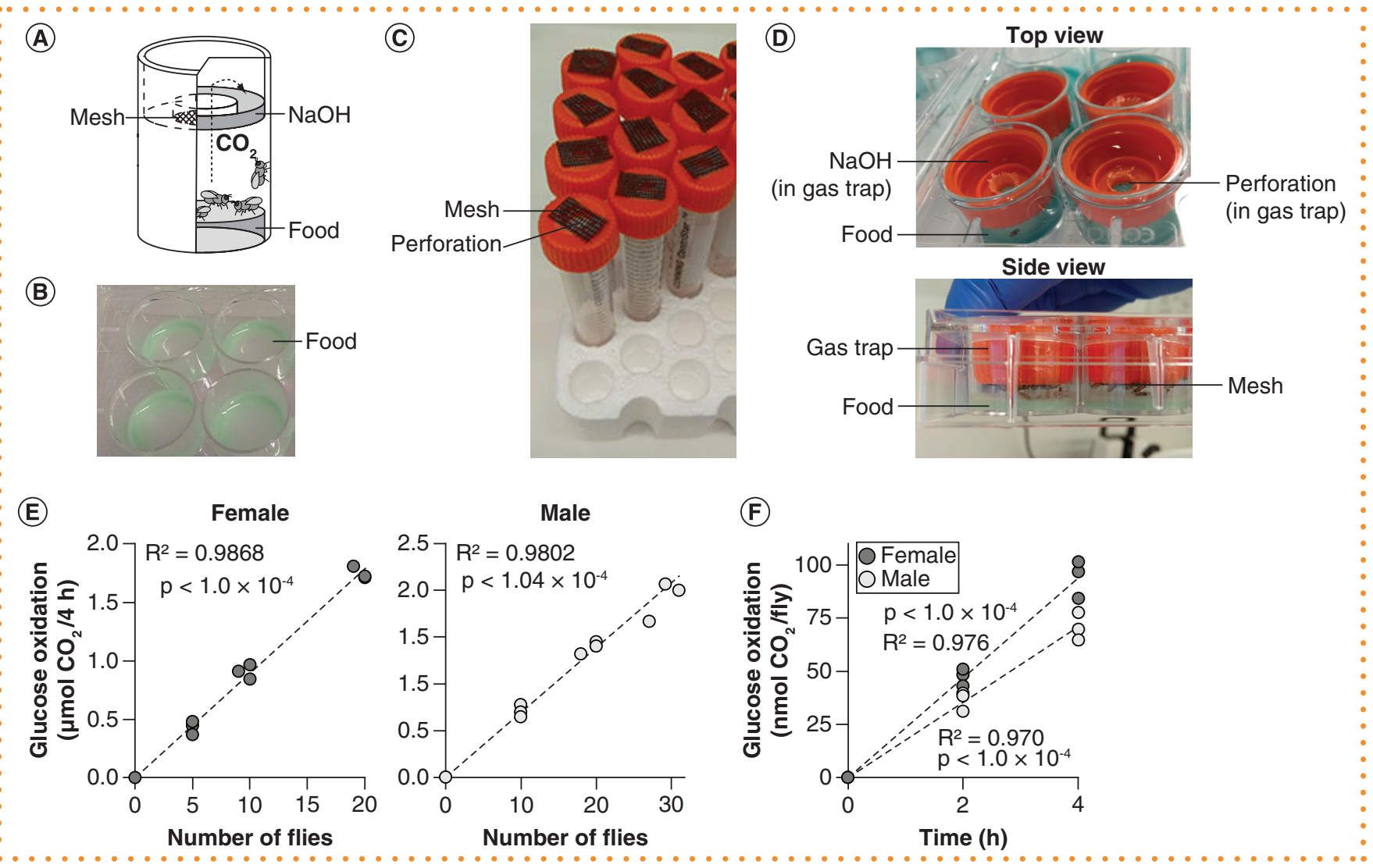

Figure 1. The Drosophila gas trap assay measures glucose oxidation in live animals. (A) Schematic of the Drosophila gas trap (details in the main text). (B-D) Photos of multiwell plates containing fly food (B), prepared Drosophila gas traps (C), and Drosophila gas traps with food and flies beneath the traps (D). (E) Different numbers of male and female flies were incubated with substrate containing uniformly ${ }^{14} \mathrm{C}$-labelled glucose (details in Supplementary protocol) and glucose oxidation was measured after $4 \mathrm{~h}$. (F) 20 male or 10 female flies were incubated as in (E) for different lengths of time prior to measuring glucose oxidation. Data in $(E \& F)$ are representative of at least two separate experiments. The correlation between glucose oxidation and fly number $(E)$ and time $(F)$ were assessed by the $R^{2}$ value and one-way ANOVA.

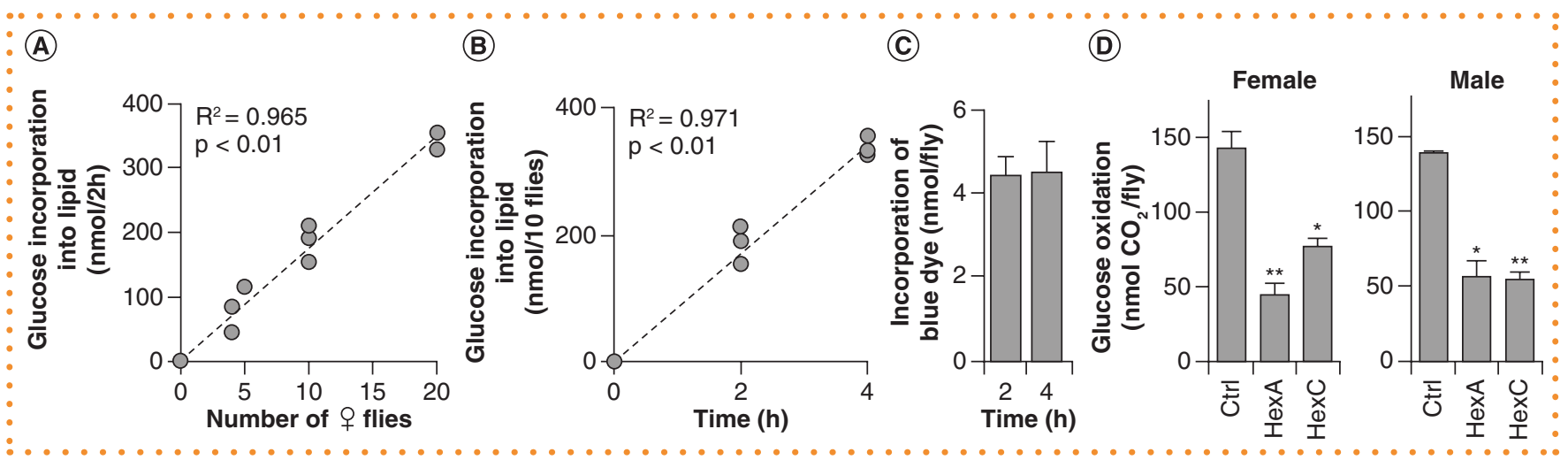

Figure 2. The Drosophila gas trap assay can be coupled with assaying other parameters and genetic manipulation. (A) The experiment was performed as in Figure $1 \mathrm{E}$, and after removing the gas-trapping solution, flies were sacrificed by freezing the entire microplate. Flies were then homogenized in a $\mathrm{CHCl} 3 \mathrm{MeOH}(2: 1)$ solution and lipids extracted using the Folch method [12]. Lipids were dried under nitrogen gas in scintillation vials, resuspended in scintillant and radioactivity was quantified by a beta-counter. (B) The experiment was performed as in Figure $1 \mathrm{~F}$ and glucose incorporation into lipid was measured as described in (A). (C) The experiment was performed as in Figure 1F. After removing the gas trap flies were homogenized in water and the absorbance was measured at $628 \mathrm{~nm}$, and compared with blue dye as a standard. (D) Control (ubi-GAL4>CGnone) flies or flies with whole body knockdown of hexokinase A (ubi-GAL4>HexA) and C (ubi-GAL4>HexC) were incubated for $3 \mathrm{~h}$ with substrate containing radiolabeled glucose (details in Supplementary protocol), after which glucose oxidation was assessed. Data in all panels are representative of at least two separate experiments. Each experiment in (C \& D) contained four separate biological replicates for each genotype, with data presented as mean + SEM. The correlation between glucose incorporation into lipid and fly number $(A)$ and time $(B)$ were assessed by the $R^{2}$ value and one-way ANOVA. Statistical significance in (C \& D) were assessed using Student's t-test. $* \mathrm{p}<0.01 ; * * \mathrm{p}<0.001$. 
- flies remained within the wells, fly-screen mesh was adhered over the hole in the gas trap (perforated tube lids) to prevent flies from escaping but still enable gaseous exchange (Figure 1C). To assay substrate oxidation, $\mathrm{NaOH}$ was added to the trap and the plate was sealed (Figure 1D). Similarly to previous methods [8], this relies on trapping diffused $\mathrm{CO}_{2}$ in an alkaline solution and thus should be just as sensitive for $\mathrm{CO}_{2}$ measurements. Our setup allows control over the diet, incubation period with the diet, and the measurement period of substrate oxidation as independent parameters, offering flexibility in the experimental design.

As proof of principle, we assessed glucose oxidation in flies. Flies were incubated with a diet containing radiolabeled glucose. We found that in both male and female flies, the amount of glucose carbon converted to $\mathrm{CO}_{2}$ was proportional to the number of flies (Figure 1E) and linear over time (Figure 1F). This enabled us to optimize the duration and number of flies in subsequent experiments.

Since this assay is nondestructive, it could enable repeated measurements of substrate oxidation over a time course and permit the collection of flies at the end of the experiment for end point metabolic measurements. For instance, glucose is not only oxidized but can be stored as triacylglycerides (TAGs). Thus, we extracted TAGs from the same flies and found that the amount of glucose incorporation into TAGs within a well increased with both fly number (Figure 2A) and linearly over time (Figure 2B). Furthermore, blue food dye was included in the food to provide visual inspection that the food mixture was homogeneous, but can also serve as a proxy of food intake. We found that a similar amount of food dye was incorporated at $2 \mathrm{~h}$ and $4 \mathrm{~h}$ (Figure $2 \mathrm{C}$ ), suggesting a steady state between food intake and dye excretion, which has been previously found to occur within a similar timeframe [11]. Overall, these experiments demonstrate that the gas trap enables substrate oxidation to be coupled with the measurement of other parameters.

The Drosophila model enables the high-throughput screening of genetic mutants. Thus, as a proof of principle, we assessed changes in glucose oxidation when hexokinase, which catalyzes the first step in glycolysis, was knocked down. We used the GAL4-UAS system [7] to generate whole-body knockdown of hexokinase $A$ (HexA) and $C$ (HexC) (Figure 2D). The flies were viable potentially due to the redundancy from the other hexokinase isoform. Despite this, knockdown of either hexokinase isoform substantially reduced glucose oxidation compared to the control flies (Figure 2D). This demonstrates that glucose incorporation into $\mathrm{CO}_{2}$ is dependent upon glucose metabolism within the flies, and that this assay can distinguish between flies of different genotypes.

Overall, this modified gas-trapping protocol provides a simple assay for highthroughput measurement of fly metabolism. It allows previous methods of gas trapping in flies [8] to be utilized in multiwell plates in the presence of labeled substrate. It enables precise control over key experimental conditions such as diet and time, and is nondestructive, thus being amenable to time-course experiments and able to be coupled to the measurement of other parameters. Furthermore, we have previously shown that this assay format can detect the oxidation of radiolabeled palmitate in cell culture [10]; the oxidation of other substrates (e.g., fatty acids, amino acids) could similarly be studied in flies. This assay ultimately provides a useful tool for merging fly genetics with radioactive tracer metabolic studies to understand the response to macronutrients on an organismal level.

\section{ACKNOWLEDGMENTS}

The authors would like to thank Daniel Fazakerley for useful discussions about the manuscript.

\section{AUTHOR CONTRIBUTIONS}

DF, JRK and DEJ conceived the study. All authors participated in experimental design. DF and JRK performed and analyzed data for the experiments. DF, JRK and DEJ wrote the manuscript. All authors edited the manuscript. DEJ supervised the study.

\section{DISCLAIMER}

The contents of the published material are solely the responsibility of the authors and do not reflect the views of the NHMRC.

\section{FINANCIAL \& COMPETING INTERESTS DISCLOSURE}

The work is supported by National Health and Medical Research (NHMRC) project grants (APP1086851 for DE James) and Diabetes Australia Research Program grants (JR Krycer). JR Krycer is recipient of the Australian Diabetes Society Skip Martin Early Career Fellowship. DE James is an NHMRC Senior Career Fellow (APP1117078). The authors have no other relevant affiliations or financial involvement with any organization or entity with a financial interest in or financial conflict with the subject matter or materials discussed in the manuscript apart from those disclosed.

No writing assistance was utilized in the production of this manuscript.

\section{OPEN ACCESS}

This work is licensed under the AttributionNonCommercial-NoDerivatives 4.0 Unported License. To view a copy of this license, visit http://creativecommons.org/licenses/ by-nc-nd/4.0/

\section{REFERENCES}

1. Burchfield JG, Kebede MA, Meoli CC et al. High dietary fat and sucrose results in an extensive and time-dependent deterioration in health of multiple physiological systems in mice. J. Biol. Chem. 293(15), 5731 (2018).

2. Fazakerley DJ, Chaudhuri R, Yang P et al. Mitochondrial CoQ deficiency is a common driver of mitochondrial oxidants and insulin resistance. eLife 7, e32111 (2018).

3. Piper MD, Skorupa D, Partridge L. Diet, metabolism and lifespan in Drosophila. Exp. Gerontol. 40(11), 857 (2005).

4. Teleman AA. Molecular mechanisms of metabolic regulation by insulin in Drosophila. Biochem. J. 425(1), 13-26 (2010).

5. Bharucha KN. The epicurean fly. using Drosophila melanogaster to study metabolism. Presented at: 2012 Sixth ACM/IEEE International Symposium Networks-on-chip. Copenhagen, Denmark, 9-12 May 2012, 1, 142-151 (2012).

6. Tennessen JM, Barry WE, Cox J, Thummel CS. Methods for studying metabolism in Drosophila. Methods 68(1), 105-115 (2014)

7. Brand A, Perrimon N. Targeted gene expression as a means of altering cell fates and generating dominant phenotypes. Development (Cambridge, England) 118(2), 401-415 (1993).

8. Bland ML. Measurement of carbon dioxide production from radiolabeled substrates in Drosophila melanogaster. J. Vis. Exp. Jove. 112, 54045 (2016).

9. Berg JM, Tymoczko JL, Stryer L. Biochemistry (5th Edition). WH Freeman, NY, USA (2002).

10. Krycer JR, Diskin C, Nelson ME, Zeng X-Y, Fazakerley DJ, James DE. A gas trapping method for high-throughput metabolic experiments. BioTechniques 64(1), 27 (2018).

11. Shell BC, Schmitt RE, Lee KM et al. Measurement of solid food intake in Drosophila via consumption-excretion of a dye tracer. Sci. Rep. 8(1), 11536 (2018).

12. Folch J, Lees M, Stanley SG. A simple method for the isolation and purification of total lipides from animal tissues. J. Biological Chem. 226(1), 497-509 (1957). 